\title{
Experiments on water droplet separation in a Ranque-Hilsch vortex tube (RHVT)
}

\author{
D. Saha ${ }^{1}$, J. C. H. Zeegers ${ }^{1}$ \& J. G. M. Kuerten ${ }^{2}$ \\ ${ }^{1}$ Applied Physics, Eindhoven University of Technology, The Netherlands \\ ${ }^{2}$ Mechanical Engineering, Eindhoven University of Technology, \\ The Netherlands
}

\begin{abstract}
We have investigated the efficiency of the Ranque-Hilsch Vortex Tube (RHVT) to separate water droplets from nearly saturated humidified $\mathrm{N}_{2}$ gas. With respect to the injected water ( $1 \%$ in mass fraction), water vapour enrichment around $15 \%$ is found at the hot exit of the RHVT where the temperature is higher than at the inlet. Measurement at the cold exit where the temperature is lower than the inlet shows an oversaturated condition, i.e., the estimated relative humidity is $\sim 200 \%$. Therefore, we measured the separation efficiency by removing the droplets as condensate from the cold side. We found that the water separation efficiency at the cold side with respect to total amount of water injected is $\sim 10 \%$ and with respect to the amount of water present at the cold side flow is $\sim 20 \%$ respectively, for cold fractions $0.3-0.7$. The condensate separation efficiency increases up to $40 \%$ by taking into account of the maximum condensable water from the oversaturated cold stream.
\end{abstract}

Keywords: Ranque-Hilsch vortex tube, droplet separation.

\section{Introduction}

The vortex tube, illustrated in Figure 1, is a device in which pressurized gas undergoes enthalpy separation. The cooling effect is attributed to the adiabatic expansion of the tangentially injected compressed gas into the cylindrical tube. The Ranque-Hilsch Vortex Tube (RHVT) consists of a tube with a typical length to diameter ratio $L / D \approx 20-50$, attached to the part referred to as the vortex chamber. Along the circumference of the vortex chamber pressurized gas is accelerated through several converging tangentially oriented nozzles and 
subsequently expanded, generating high swirl motion, i.e., the vortex. There exists a radial pressure gradient due the swirling motion of the gas flowing through the tube and energy is transported from the core towards the periphery of the tube [1]. Because of the energy transport, radial temperature separation takes place, i.e., the core of the flow cools down and the peripheral region heats up [2]. The expanded gas splits into two streams which leave the system through two exits on either side of the vortex chamber. A fraction of the gas from the inner core having lower temperature than the inlet leaves the device through the exit closer to the vortex chamber which has a smaller diameter than the main tube. This opening is termed the cold exit. The remaining part of the gas flows along the tube periphery with a higher temperature than the inlet to the exit at the end of the main tube. This outlet is referred to as the hot exit.

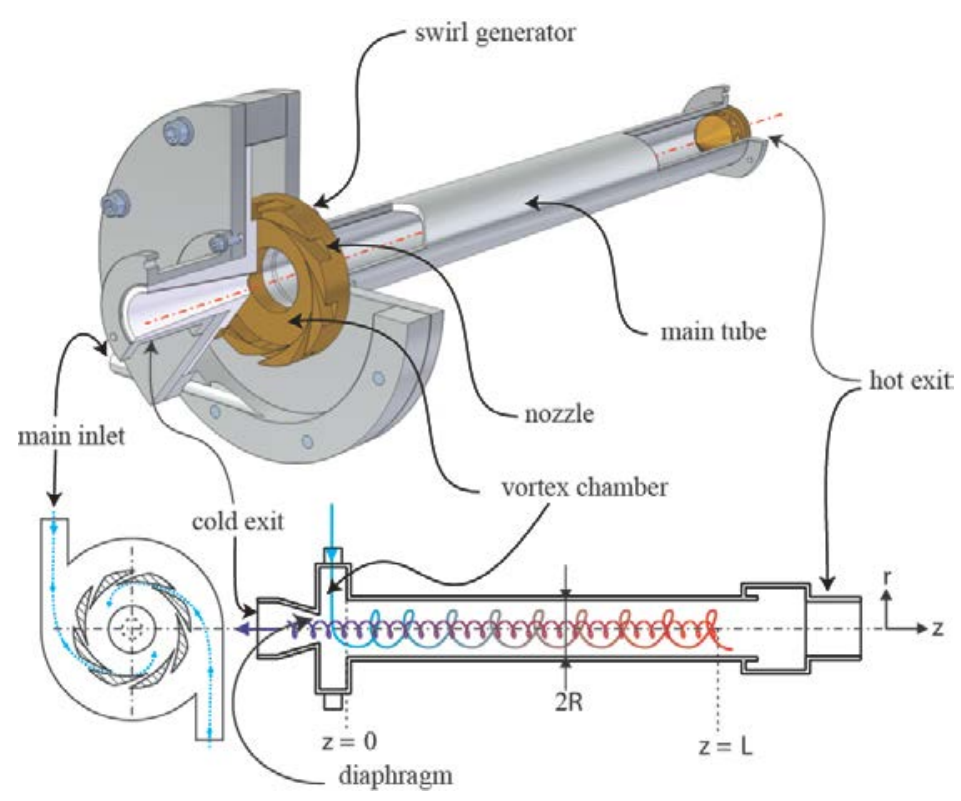

Figure 1: Ranque-Hilsch vortex tube with the swirl generator having eight converging nozzles. The schematic shows how the inflow splits into two streams of two different temperatures, i.e., hot and cold streams which leave the vortex tube through two exits.

The main focus of the research on the RHVT has been on the energy separation process, specifically to upgrade its thermal efficiency. In this study we investigate a second possible application of the RHVT, namely condensation and separation. The swirl velocity of the gas at the inlet of the RHVT can reach up to the sonic velocity resulting in high centrifugal acceleration, which can be used to separate condensable components in the form of liquid droplets. The cooling effect of the RHVT can be used to liquefy condensable components in the gas, such as $\mathrm{CO}_{2}$. We make use of the high swirl intensity and the cooling effect to 
separate water droplets from the carrier $\mathrm{N}_{2}$ gas to investigate the feasibility of the RHVT as a separator for the removal of condensable components from a gas stream.

\section{Experimental setup}

The experimental setup is schematically illustrated in Figure 2. Pressurized $\mathrm{N}_{2}$ ( $\sim 6$ bar) is preheated to $65^{\circ} \mathrm{C}$ by a heater (Enon, Vulcanin SA) prior to flowing into the humidifying vessel where $\mathrm{N}_{2}$ is mixed with a spray of water droplets. The volume of the humidifying vessel is $0.44 \mathrm{~m}^{3}$ with a diameter of $60 \mathrm{~cm}$ and a height of $1.6 \mathrm{~m}$. Two high pressure water nozzles (Danfoss) with an opening of $0.3 \mathrm{~mm}$ and a spray angle of $45^{\circ}$ are placed inside the vessel. A high pressure water pump (Nessie Plug \& Play ${ }^{\mathrm{TM}}$ ) with a capacity of $75 \mathrm{l} / \mathrm{hr}$ at 100 bar was used to inject the water droplets into the humidifier. A water filled container is placed on a digital scale (Kern FKB36K0.2, max $36.1 \mathrm{~kg}$, reproducibility $0.2 \mathrm{~g}$ ) which is connected to a PC enabling a continuous measurement of the water consumption in time. The relative humidity is measured by capacitive humidity probes (MDR3 General Electric) with an accuracy of $2 \%$ for the range of $0 \% \leq$ $\mathrm{RH} \leq 90 \%$ and $3 \%$ for $90 \% \leq \mathrm{RH} \leq 100 \%$. Temperature is measured by the humidity probe which has a built in temperature sensor with an accuracy of $0.5 \mathrm{~K}$. Digital manometers (Keller) are used to measure the pressure which has an accuracy of $0.02 \%$ for the range of $0-21$ bar. The mass flow rate is measured by coriolis mass flow sensors (KROHNE OPTIMASS 6400C S10 and 6400C S25) which have an accuracy of $\pm 0.35 \%$ of the actual measured flow rate. A parameter referred to as the cold fraction, $\varepsilon=\mathrm{m}_{\mathrm{c}} / \mathrm{m}_{\mathrm{in}}$, where $\mathrm{m}_{\mathrm{c}}$ is the cold exit mass flow rate and $m_{\text {in }}$ is the inlet mass flow rate, represents the mass flow rate through the exits of the RHVT. The separation experiments are performed with two different types of swirl generator. One has eight converging, tangentially oriented nozzles along the periphery with a size of $1 \times 14 \mathrm{~mm}$ and the other has two conical nozzles at $45^{\circ}$ from each other (see Figure 3 ). The RHVT used in the experiment has a main tube length of $L=1.4 \mathrm{~m}$ and inner diameter of $40 \mathrm{~mm}$. The vortex chamber has an inner diameter of $80 \mathrm{~mm}$ for both swirl generators. The mass flow rate was kept constant at $252 \mathrm{~kg} / \mathrm{hr}$ for all experiments.

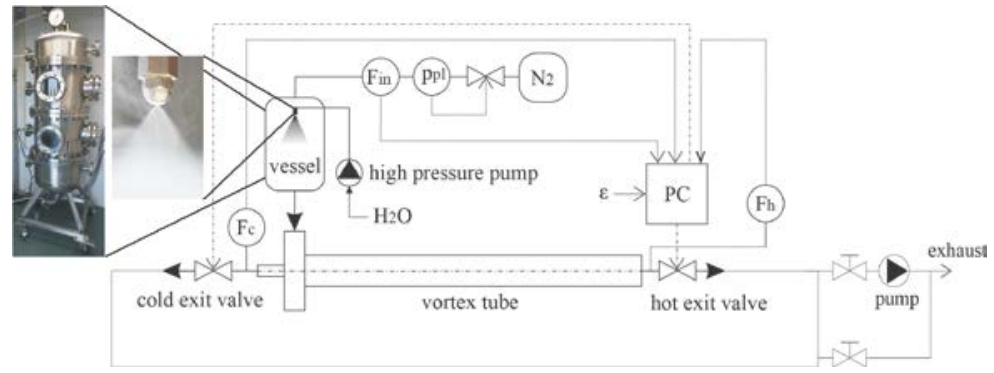

Figure 2: Schematic overview of the experimental setup. Laboratory view of the humidifying vessel and the high pressure water nozzle are shown aside. 

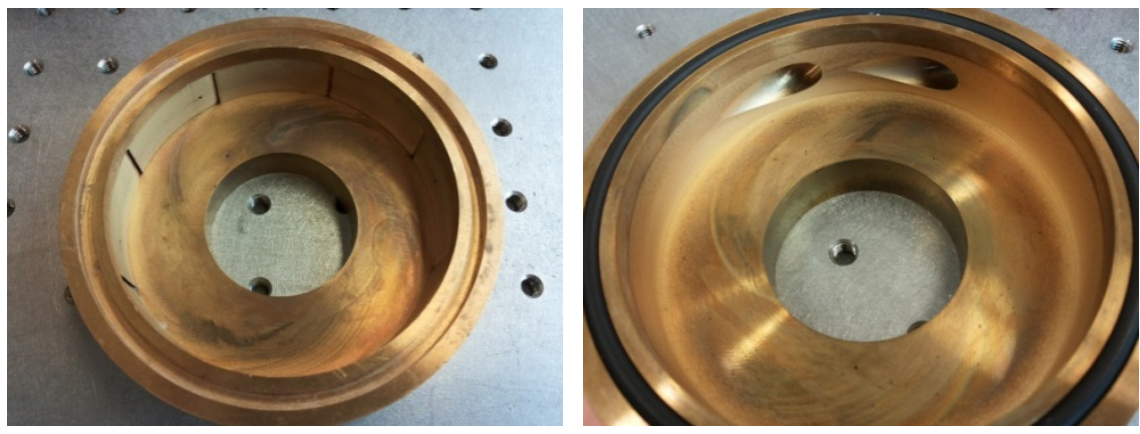

Figure 3: Two types of swirl generator. One (left) has eight rectangular converging nozzles and the other (right) has two conical nozzles.

\section{Results and discussion}

The mass flow rates of water in the inlet and at the two exits determine the separation efficiency of the RHVT. In this humidity study we used a quantity referred to as the mixing ratio to determine the amount of water in the gas mixture. The mixing ratio, $\mathrm{MR}$, is the mass ratio of the water to the $\mathrm{N}_{2}$ gas and defined as [3]

$$
M R=\frac{p_{\text {sat }}(T)}{p-p_{\text {sat }}(T)} \frac{M_{\mathrm{H}_{2} \mathrm{O}}}{M_{N_{2}}}
$$

Here $p_{\text {sat }}(T)$ is the saturated vapour pressure at the measured temperature, $p$ is the measured pressure and $M_{H 2 O}$ and $M_{N 2}$ are the molar masses of the water and $\mathrm{N}_{2}$ gas respectively. The injection rate of the water is deduced rom the rate at which the digital scale changes. Therefore, the amount of water in the $\mathrm{N}_{2}$ gas per unit time is precisely known.

The water mass balance in the RHVT can be written as

$$
M R_{p l}=\varepsilon M R_{\text {cold }}+(1-\varepsilon) M R_{\text {hot }}+\delta M R
$$

where $M R_{\text {cold }}$ and $M R_{\text {hot }}$ are the mixing ratio of the cold and the hot exit respectively and $\delta M R$ is the measurement uncertainty involved during the experiment. It is found that the mean error in mass balance for $\varepsilon=0.1-0.9$ is $\sim 8 \%$.

The separation efficiency of the water vapour at the hot exit of the RHVT is defined as

$$
\eta_{v}=\left(\frac{M R_{h}}{M R_{p l}}-1\right) \times 100 \%
$$

It is a measure of the mass of water present in the hot stream at the hot exit of the RHVT compared to the mass of water at the inlet. 


\subsection{Separation at the hot side}

In the first experiment, the plenum relative humidity was kept constant at $98 \%$ at a plenum temperature of $38^{\circ} \mathrm{C}$, the plenum pressure was $5.0-5.3$ bar and the mass flow rate of $\mathrm{N}_{2}$ was $252 \mathrm{~kg} / \mathrm{hr}$. The cold fraction was varied from $0.05 \%$ to $0.95 \%$. The mean mixing ratio of the plenum and the hot exit for all cold fractions is shown in Figure 4 (left). The mixing ratio at the hot exit is larger than that of the plenum for cold fractions larger than $\varepsilon=0.3$, resulting in vapour enrichment. A maximum separation of $\sim 15 \%$ occurs at $\varepsilon \sim 0.85$. The physical mechanism of this enrichment is probably due to an abundance of condensate that occurs in the center of the vortex tube, that is centrifuged towards the wall.
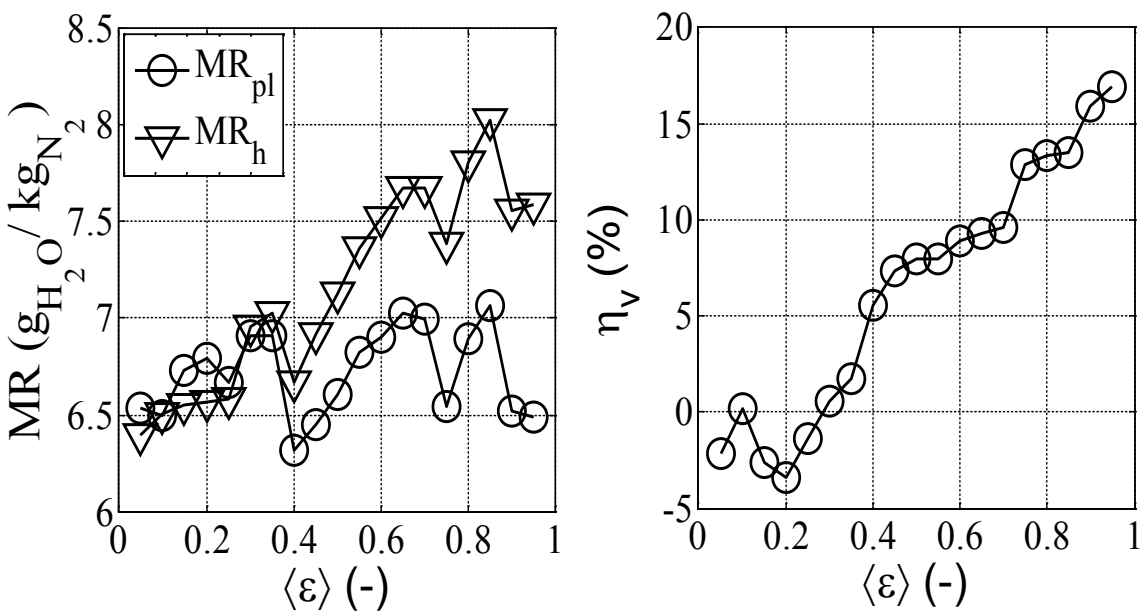

Figure 4: The mean mixing ratio of the plenum (open circles) and the hot exit triangles) as a function of the cold fraction, $\varepsilon$ (left). The vapour enrichment (on the right) as a function of the cold fraction.

Near the wall, the re-evaporated vapour then flows towards the hot side. When the physical separation in the vortex tube is constant, it would be obvious that at increased cold fraction, $\varepsilon$ the enrichment at the hot side will increase as per definition. In the second experiment the swirl generator was replaced by the one having only two nozzles to investigate the effect of vortex wobbling on droplet separation. Using the current swirl generator, analysis of Laser Doppler Anemometry (LDA) experiments shows that the velocity varies periodically in time with high amplitude. The observed presence of higher harmonics in the velocity signal in radial and axial direction is attributed to the fact that the axis of the main vortex is not aligned with the geometric axis of the tube; i.e., the vortex precesses around the RHVT axis, a phenomenon called vortex wobbling. The flow rate, plenum relative humidity and the plenum pressure were kept the same as in the previous experiment to compare the results. In this experiment the cold fraction, $\varepsilon$ was varied stepwise with an interval of 0.1 . Figure 5 shows no 
remarkable improvement in separation efficiency and hence there is no influence of vortex wobbling on separation. The agreement between the two experiments reflects that separation sets in at about $\varepsilon \sim 0.3$ and increases with increasing cold fraction. In conclusion it is clear that for $\varepsilon>0.8$, a hot side vapour enrichment of $10-15 \%$ can be achieved. But at $\varepsilon>0.8$, the hot side stream is per definition is already quite small. So the efficient upgrade of the total mass flow of water as vapour in terms of enrichment is insignificant. We replaced the brass tube with a transparent glass tube to visualize the events taking place at the hot side. We noticed streamlines of water swirling along the tube wall where the temperature is higher than at the core, which disappeared when they approach the exit. We observed two distinct features. The amount of water is very low and the swirling flow patch is not continuous. These two observations could possibly explain the reason for the low separation performance at the hot side.

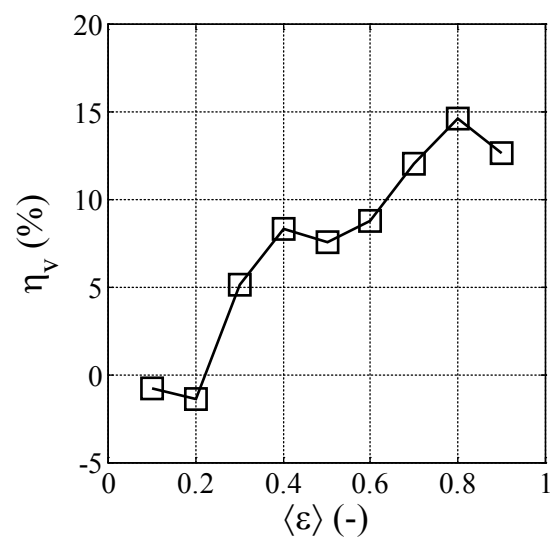

Figure 5: The vapour enrichment at the hot side as a function of the cold fraction using the nozzle having two holes.

\subsection{Separation at the cold side}

Since the separation of the water droplets at the hot side in the RHVT has been shown to be small, we continue to investigate the separation performance at the cold side instead of the hot side of the vortex tube, i.e., removing water from the gas stream in the form of condensate. Figure 6 (left) shows the relative humidity at the plenum, hot and cold side of the vortex tube in time at a constant plenum temperature of $36^{\circ} \mathrm{C}$. Figure 6 (right) shows the temperature in time for different cold fractions. For a constant plenum humidity of $\sim 75 \%$, the relative humidity at the hot side increases slowly with increasing flow rate through the hot exit (lower cold fraction), i.e., for $10-90 \%$ of the flow through the hot exit the relative humidity increases by a factor two with respect to the initial relative humidity of $\sim 10 \%$ at the hot side. On the contrary, at a cold fraction of $\varepsilon=0.1-0.6$, the relative humidity at the cold side rapidly increases towards oversaturation. The humidity sensor at oversaturation ceases to function as 
indicated by an abrupt drop in the signal to a negative value. This could imply the presence of water droplets at the cold side at $\sim 20^{\circ} \mathrm{C}$ for $10-60 \%$ of the inflow through the cold exit. Therefore, a separator to remove the droplets from the cold exit has been designed as shown in Figure 7. Since the humidity sensor is unable to measure the relative humidity for the range of cold fraction $0.1-0.6$ as shown in Figure 6, based on the assumption that the partial vapor pressure is constant, we approximated the relative humidity at the cold side using Antoine's equation relating vapor pressure to the temperature, which indicates the over saturated state at the cold side leading to condensation for a cold fraction $0.1-0.8$. This supports the effort to remove the droplets by the newly designed separator for the cold side as shown in Figure 7. Figure 8 (left) shows the total amount of water injected into the gas (black circles) and the separated amount from the cold side by two different separators (circles in blue and red).
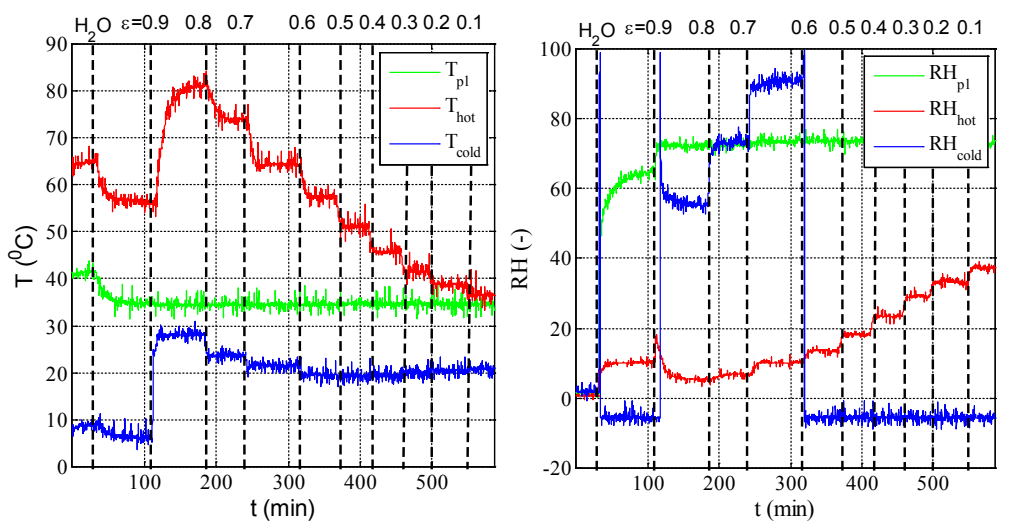

Figure 6: Relative humidity (left) and the temperature (right) of the plenum, hot and cold side in time (bottom scale) for different cold fractions, $\varepsilon$ (upper scale).

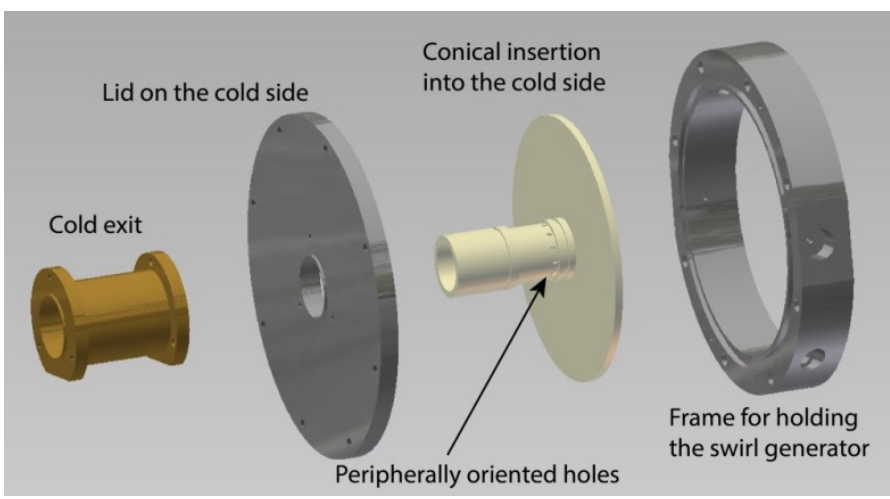

Figure 7: Holes are drilled around the periphery of the cold exit to remove the water droplets from the cold side of the RHVT. 


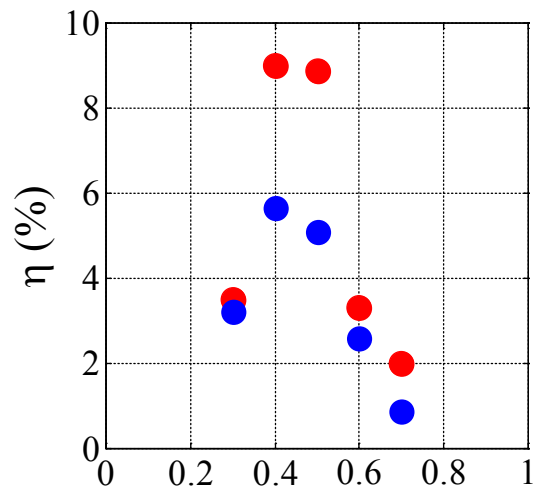

$\langle\varepsilon\rangle(-)$

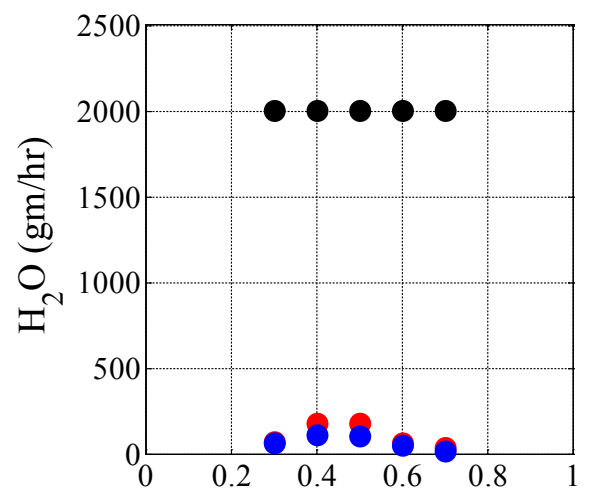

$\langle\varepsilon\rangle(-)$

Holes $5 \mathrm{~mm} 15 \mathrm{~mm}$ downstream of cold exit $\bigcirc$ injected $\mathrm{H}_{2} \mathrm{O}$.

Figure 8: Total amount of water injected into the gas for cold fractions $0.3-0.7$ (black circles) and the amount of water extracted by two different types of separators at the cold side (circles in blue and red) (left). Separation efficiency of the cold side with respect to the total amount of water in the plenum gas mixture (right).

Figure 8 (right) shows the corresponding efficiency as the ratio of total amount of water injected into the gas stream to the amount separated by the separators. For both separators, the maximum amount of water is extracted at a cold fraction of 0.4 but the efficiency obtained is insignificant, i.e., $\sim 10 \%$. One of the reasons for this low efficiency is the fact that part of the injected water is also present in the hot stream as vapor and flowing out of the hot exit. Therefore, we calculate the cold side efficiency as the ratio of the amount extracted by the separators to the cols side water content. Figure 9 (left) shows a linear increase in water content in the cold stream (black circles) with an increase of the cold fraction and the amount of water separated (blue and red circles). Figure 9 (right) shows the corresponding efficiency. The result shows that the condensate removal efficiency increases to $\sim 20 \%$ for both types of separators. Since the cold stream is over saturated, we calculate the maximum condensable amount of water (black circles) from over saturated cold stream as shown in Figure 10 (left, black circles). Based on the ratio of the water separated by the separator (circles in red and blue) to the maximum condensable amount of water, we found the maximum of the separator efficiency, at the cold fraction of $\varepsilon=0.4$, to be $\sim 40 \%$ for the one attached closer to the exit, i.e., the one with the holes $5 \mathrm{~mm}$ away from the exit as shown in Figure 10 (right, red circles). This increase in efficiency gives an insight of the quality of the separator and the possibility to enhance the condensate removal by optimizing the separator design. 


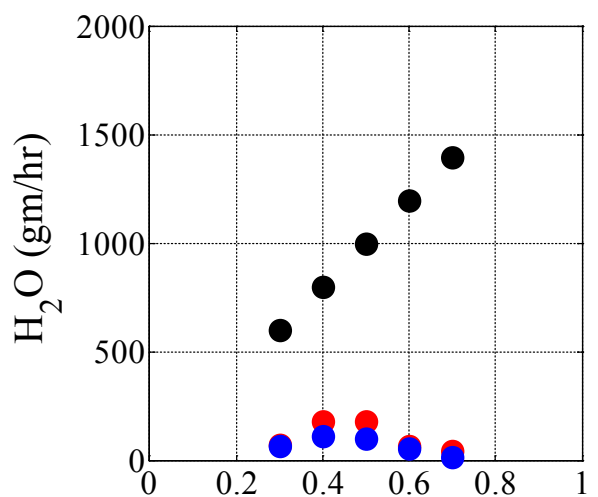

$\langle\varepsilon\rangle(-)$

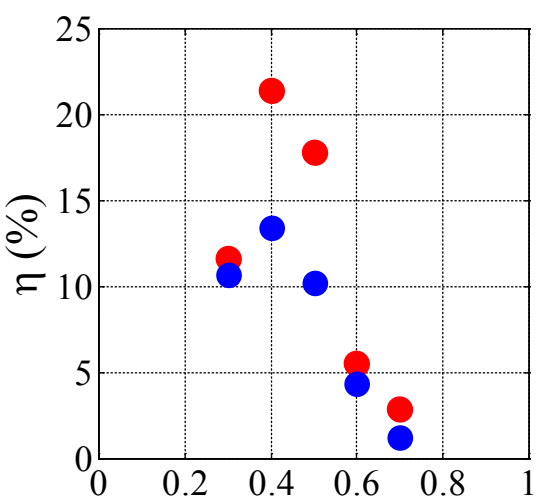

$\langle\varepsilon\rangle(-)$

Holes $5 \mathrm{~mm} 15 \mathrm{~mm}$ downstream of cold exit $\mathrm{H}_{2} \mathrm{O}$ in cold exit.

Figure 9: Amount of water flowing to the cold side (black circles) and the amount extracted by the separators (left) and the corresponding efficiency (right).

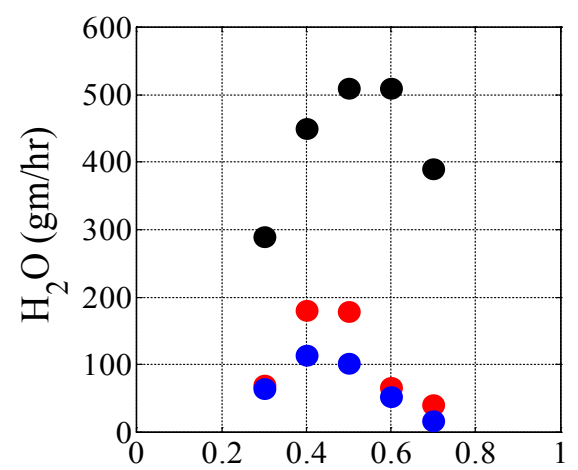

$\langle\varepsilon\rangle(-)$

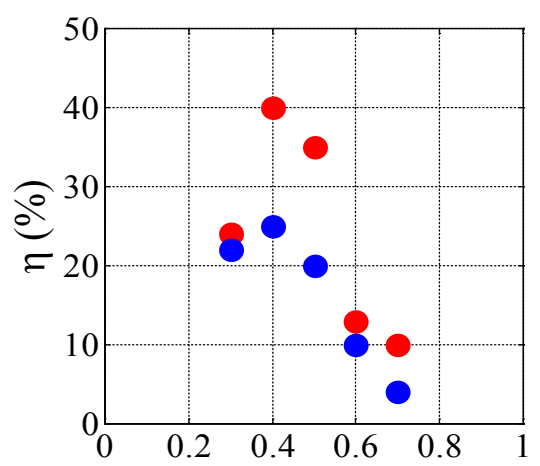

$\langle\varepsilon\rangle(-)$

Holes $5 \mathrm{~mm} 15 \mathrm{~mm}$ downstream of cold exit $\max$ sep. $\mathrm{H}_{2} \mathrm{O}$.

Figure 10: Maximum amount of condensable water at the cold stream (black circles) and the amount of water that has been removed by the separators (left) and the corresponding efficiency (right).

\section{Conclusions}

In this experimental investigation, a feasibility study of the capabilities of the Ranque-Hilsch vortex tube as a separator, to remove water droplets from $\mathrm{N}_{2}$ gas has been carried out. First, we investigated the vapour enrichment at the hot side, where the temperature is higher than the plenum temperature, as the preferred exit for removing water vapour. The results from two different sets of experiments where two types of swirl generators were used show a similar 
separation efficiency. The maximum vapour enrichment at the hot exit is found to be $\sim 25-20 \%$ for a cold fraction of $\varepsilon \sim 0.8$. We studied the influence of the vortex wobbling on droplet separation in the tube and found that the separation efficiency is independent of the wobbling. We conclude that, as the hot side contains water vapour, the low separation efficiency is insignificant for potential applications. We carried out a new separation experiment at the cold side with the objective to remove water as condensate instead of vapour. We devised a preliminary design for the separator with holes around the periphery of the cold exit. We found that, for the cold fraction of $\varepsilon=0.3-0.7$, the water separation efficiency at the cold side with respect to the amount of water present at the cold side flow is $\sim 20 \%$. We found the condensate separation efficiency as high as $40 \%$ by taking into account of the maximum condensable water from the oversaturated cold stream. This result indicates further scope to improve the design of the separator and obtain higher condensate removal efficiencies which could be of potential benefit for the industrial applications.

\section{Acknowledgement}

This research is supported by the Dutch Technology Foundation, STW.

\section{References}

[1] Eiamsa-Ard, S. \& Promvonge, P. (2008). Review of Ranque-Hilsch effects in vortex tubes. Renew. Sust. Energ. Rev., 12 1822-1842. 7,112,119.

[2] Xue, Y., Arjoumandi, M. \& Kelso, R. (2010). A critical review of temperature separation in a vortex tube. Exp. Therm. Fluid Sci., 34 13671374. 7, 112.

[3] Liew, R. (2013). Droplet behaviour and thermal separation in RanqueHilsch vortex tube. PhD thesis. 